\title{
Pump photons present in a non-linear process as a witnesses of non-classicality of a system
}

\author{
Priyanka $^{a}$, Savita Gill ${ }^{b}$ \\ University Institute of Engineering and Technology, Kurukshetra University Kurukshetra, 136119, India \\ ${ }^{a}$ Chauhan7101@gmail.com, ${ }^{b}$ Savita2015@kuk.ac.in
}

Corresponding author: Savita Gill, Savita2015@kuk.ac.in

ABSTRACT We have studied non-classical effects, i.e. higher order photon antibunching (HOA) and higher order sub-Poissonian photon number statistics (HOSPS) in various non-linear optical processes like second harmonic generation, fourth harmonic generation, coherent anti-Stokes Raman scattering (CARS) and coherent anti-Stokes hyper-Raman scattering (CAHRS) using short time interaction techniques. The non-classical effects directly depend on number of photons prior to interaction with non-linear medium has already been studied but we have found that non-linear processes involving equal number of pump photons have same higher order photo antibunching (HOA) and higher order sub-Poissonian photon number statistics (HOSPS) independent of the non-linear process involved.

KEYWORDS Higher order sub-Poissonian photon number statistics, higher order photon antibunching, optical processes.

FOR CITATION Priyanka, Savita Gill Pump photons present in a non-linear process as a witnesses of nonclassicality of a system. Nanosystems: Phys. Chem. Math., 2022, 13 (1), 71-77.

\section{Introduction}

Non-classical states cannot be characterized by a mixture of coherent states and these are often defined by the negative values of Glauber-Sudarshan P-functions [1,2]. In fact, there is no method for determining the P-function experimentally, except for a single proposal [3]. A number of operational criteria for witnessing non classicality have been developed [4,5]. These observers of non-classicality can be communicated as the moments of creation and annihilation operators and a nonclassical property seen through a moment-based model that observes a non-classical characteristic through a lower order connection is known as a lower-order non-classical property. Higher-order non-classicality, as the name implies, refers to the non-classical characteristics revealed by higher order correlations. Higher order photon antibunching (HOA) [6], higher-order sub-Poissonian photon number statistics (HOSPS) [7,8], higher order squeezing of Hong-Mandel type [9,10] are the most often researched higher order non-classical characteristics. All of these non-classical characteristics have lower order equivalents that have been well investigated [11,12]. Due to the successful experimental characterizations of higher-order non-classical states, much attention has been given to these states recently [13-16]. The fact that weak non-classicalities not identified by their lower order equivalents can be recognized by higher order non-classicality criteria has led to a significant number of theoretical studies as well $[14,15]$. Indeed, higher order photon antibunching (HOA) and higher order squeezing (HOS) has been accounted in an opto-mechanical like system [17], finite dimensional coherent state [5], optical coupler [18], hyper-Raman process [19] and higher order sub-Poissonian photon number statistics (HOSPS) has been accounted in finite dimensional coherent state [5], photon added and subtracted squeezed coherent states [20]. Previously, research into these non-classical phenomena was mostly for academic interest [21], but their numerous applications in quantum information theory, such as optical communication [14], dense coding [22], quantum teleportation [23], and quantum cryptography [24], are now well-known. Non-classicality has been shown to be a required input for the entangled state [25]. All of the physical systems mentioned above are experimentally feasible and may be easily seen in a non- linear optics laboratory $[26,27]$. Photon number statistics may be obtained experimentally using the homodyne detection technique [28,29]. A number of new opportunities for non-linear optics have arisen due to the rapid growth of nanotechnology and nanoscience. During the last few decades, non-linear optical materials have made significant progress in laser technology and these materials have large non-linear optical properties and a fast non-linear response for various photonic applications such as pulsed laser deposition, laser ablation, optical information processing, optical communication, optical limiters and optical data storage [30-35]. Nanomaterials with large non-linear responses are useful in photocatalysis and optical limiting applications [36] and optical non-linear microscopy [37]. Materials with large third order optical non-linearity and fast response time will be required for future optical device applications [38,39]. Due to their high non-linear optical response of these nonlinear optical materials, these materials are used in fiber optic communication systems such as all digital signal restoration, routing units, de- multiplexing and multiplexing and optical storage media [39] as well as in optical switching [40]. Strong optical non-linearities observed due to the quantum

(c) Priyanka, Savita Gill, 2022 
confinement effects such as second and third order optical non-linearities and non-linear optical absorption that can be studied for making laser second and third harmonic generators and optical modulators [38,39]. There has been a lot of research done on measuring third order nonlinear susceptibility $\chi^{3}$ to examine optical nonlinearity of nanoparticles may be $\mathrm{Z}$ scan technique and degenerate four wave mixing experiments [39]. Hanamura analyzed theoretically the third order optical polarizability $\chi^{3}$ and the oscillator strength in semiconductor microcrystallites [41].

The state of non-classicality of the non-linear optical system is described in segment 2 of the current study. Segment 3 will introduce a second order solution of equation of motion by means of an illustration of fourth harmonic generation process and show the presence of higher order photon antibunching (HOA) and higher order sub-Poissonian photon number statistics (HOSPS). In segment 4, we have investigated the presence of higher order non-classical effects in second harmonic generation, coherent anti-Stokes Raman scattering process (CARS) and coherent anti-Stokes hyperRaman scattering (CAHRS) non-linear optical processes, as well as their direct association with pump photons present in the system. In section 5, we used graphs to compare the results and section 6 is devoted to the conclusion.

\section{Condition of non-classicality of a non-linear optical system}

\subsection{Condition for higher order photon antibunching(HOA)}

Lee presented the higher order photon antibunching (HOA) criteria as follows [42]:

$$
R(l, p)=\frac{\left\langle N_{x}^{l-1}\right\rangle\left\langle N_{x}^{p+1}\right\rangle}{\left\langle N_{x}^{l}\right\rangle\left\langle N_{x}^{p}\right\rangle}-1<0
$$

where number operator is represented by $N$.

$\left\langle N^{(k)}\right\rangle=\langle N(N-1)(N-2) \cdots(N-k+1)\rangle$ is the $k^{t h}$ factorial moment of the number operator. Integers $l$ and $p$ fulfilling condition $l \leq p \leq 1$ and $x$ addendum indicates specific mode. $p=1$ is picked by Ba An [43] and condition of the $l^{t h}$ order photon antibunching is reduced to

And:

$$
A_{x, l}=\frac{\left\langle N_{x}^{l+1}\right\rangle}{\left\langle N_{x}^{l}\right\rangle\left\langle N_{x}\right\rangle}-1<0
$$

$$
\left\langle N_{x}^{l+1}\right\rangle\left\langle\left\langle N_{x}^{l}\right\rangle\left\langle N_{x}\right\rangle\right.
$$

Physically, a state which is photon antibunched in the $l^{\text {th }}$ order must be photon antibunched in the $(l-1)^{t h}$ order. Therefore, we can simplify (3) as:

$$
\left\langle N_{x}^{l+1}\right\rangle<\left\langle N_{x}^{l}\right\rangle\left\langle N_{x}\right\rangle<\left\langle N_{x}^{l-1}\right\rangle\left\langle N_{x}^{2}\right\rangle<\cdots<\left\langle N_{x}\right\rangle^{l+1},
$$

and obtain condition of $l^{\text {th }}$ order photon antibunching as:

$$
d(l)=\left\langle N_{x}^{l+1}\right\rangle-\left\langle N_{x}\right\rangle^{l+1}<0 .
$$

From equation (4), we can see that for sub-Poissonian state $d(l)<0$. Along these lines, we can say that a single photon source utilized in quantum cryptography should fulfill the criteria given in equation (4) of higher order photon antibunching (HOA).

\subsection{Criteria for higher order sub-poissonian photon number statistics (HOSPS)}

Mishra and Prakash [44] provide the criteria of $(l-1)^{t h}$ order higher order sub-Poissonian photon number statistics (HOSPS) is given as:

$$
D(l-1)=\sum_{k=0}^{l} \sum_{i=0}^{l-k}{ }^{l} C_{k}(-1)^{k} S_{2}(l-k, i)\left\langle N^{i}\right\rangle\langle N\rangle^{k}-\sum_{k=0}^{l} \sum_{i=0}^{l-k}{ }^{l} C_{k}(-1)^{k} S_{2}(l-k, i)\langle N\rangle^{k+i}<0,
$$

where $S_{2}(l, k)$ is the second-order Stirling number. For $l=3$, the condition of second order sub-Poissonian photon number statistics (HOSPS) is described as:

$$
D(2)=\left\langle N^{3}\right\rangle+2\langle N\rangle^{3}-3\left\langle N^{2}\right\rangle\langle N\rangle+3\left\langle N^{2}\right\rangle-3\langle N\rangle^{2}<0 .
$$

\section{Fourth harmonic generation process}

To examine higher order photon antibunching (HOA) and higher order sub-Poissonian photon number statistics (HOSPS), we used the fourth harmonic generation process, which involves absorption of four photons, each with a frequency $\omega_{1}$ and the emission of one photon with frequency $\omega_{2}$ where $\omega_{2}=4 \omega_{1}$. For this process, the Hamiltonian is:

$$
H=\omega_{1} a^{\dagger} a+\omega_{2} b^{\dagger} b+g\left(a^{4} b^{\dagger}+a^{\dagger 4} b\right),
$$

where $a^{\dagger}(a), b^{\dagger}(b)$ are the creation (annihilation) operators and g is coupling constant. $A=a \exp i \omega_{1} t, B=b \exp i \omega_{2} t$ are the gradually varying operators at frequencies $\omega_{1}$ and $\omega_{2}$. 


\subsection{Time evolution of pump mode $A$}

The Heisenberg equation of motion for the time evolution of operator in mode $\mathrm{A}$ is given as:

$$
\frac{d A}{d t}=\frac{\partial A}{\partial t}+i[H, A]
$$

We obtain:

$$
\dot{A}=-4 i g A^{+3} B
$$

and

$$
\dot{B}=-i g A^{4} \text {. }
$$

We now expand $A(t)$ using Taylor series expansion and treating terms up to $g^{2} t^{2}$ by using short time approximation as:

$$
A(t)=A-4 i g t A^{\dagger 3} B+2 g^{2} t^{2}\left(12 A^{\dagger 2} A^{3} N_{B}+36 A^{\dagger} A^{2} N_{B}+24 A N_{B}-A^{\dagger 3} A^{4}\right) .
$$

Using equation (11), number operator $N_{A}(t)=A^{\dagger}(t) A(t)$ is given as:

$$
N_{A}(t)=A^{\dagger} A-4 i g t\left(A^{\dagger 4} B-A^{4} B^{\dagger}\right)+4 g^{2} t^{2}\left(16 A^{\dagger 3} A^{3} N_{B}+72 A^{\dagger 2} A^{2} N_{B}+96 A^{\dagger} A N_{B}+24 N_{B}-A^{\dagger 4} A^{4}\right) \text {. }
$$

To investigate photon antibunching, we start with a quantum state that is the product of coherent state $|\alpha\rangle$ for pump mode $\mathrm{A}$ and vacuum state $|0\rangle$ for stokes mode B i.e.:

$$
|\psi\rangle=|\alpha\rangle_{A}|0\rangle_{B}
$$

Using equation (13) in equation (12), we get average value of $\left\langle N_{A}(t)\right\rangle_{\alpha}=\left\langle A^{\dagger}(t) A(t)\right\rangle$ as:

$$
\left\langle N_{A}(t)\right\rangle_{\alpha}=|\alpha|^{2}-4 g^{2} t^{2}|\alpha|^{8}
$$

where $A|\alpha\rangle=\alpha|\alpha\rangle$. Now, the average value of $N_{A}^{4}(t)$ is given as:

$$
\left\langle N_{A}^{4}(t)\right\rangle_{\alpha}=\left\langle A^{\dagger 4}(t) A^{4}(t)\right\rangle=|\alpha|^{8}-8 g^{2} t^{2}\left(2|\alpha|^{14}+9|\alpha|^{12}+12|\alpha|^{10}+3|\alpha|^{8}\right) .
$$

Now, using equations (14) in equation (4), we get third order photon antibunching as

$$
d_{A}(3)_{\alpha}=-24 g^{2} t^{2}\left(3|\alpha|^{12}+4|\alpha|^{10}+|\alpha|^{8}\right) .
$$

Equation (15) shows that fourth harmonic generation process satisfies the criteria of higher order photon antibunching.

To examine higher order photon antibunching, we used the initial state which is equal to the product of vacuum state $|0\rangle$ for pump mode $\mathrm{A}$ and $|\beta\rangle$ for stokes mode $\mathrm{B}$ i.e.:

$$
|\psi\rangle=|0\rangle_{A}|\beta\rangle_{B}
$$

Now, taking average values of $N_{A}^{4}(t)$ in pump mode A with respect to condition (16) is given as:

$$
\left\langle N_{A}^{4}(t)\right\rangle_{\beta}=0 .
$$

Using equation (17) in equation (4), we obtain third order photon antibunching in mode A in relation to $|0\rangle|\beta\rangle$ is given as:

$$
d_{A}(3)_{\beta}=0 \text {. }
$$
$|0\rangle|\beta\rangle$.

Equation (18) shows that higher order photon antibunching is absent in mode A with respect to the quantum state

Now, using equations (12, 13 and 16) in equation (6), we obtain:

$$
\begin{gathered}
D(2)_{\alpha}=-60 g^{2} t^{2}|\alpha|^{8}, \\
D(2)_{\beta}=0,
\end{gathered}
$$

we obtain a negative value in equation (19), which indicates that higher order sub-Poissonian photon number statistics exists in mode A with respect to quantum state $|\psi\rangle=|\alpha\rangle_{A}|0\rangle_{B}$.

\section{Non-classicality in other non-linear optical processes}

We have analyzed higher order photon antibunching (HOA) and higher order sub-Poissonian photon number statistics (HOSPS) in various non-linear optical processes and all the results that we have obtained is mentioned in Table 1 and Table 2 respectively.

In Table 1 and Table 2, * represents the average values are taken with regards to $|\alpha\rangle|0\rangle|0\rangle$ in mode A. 


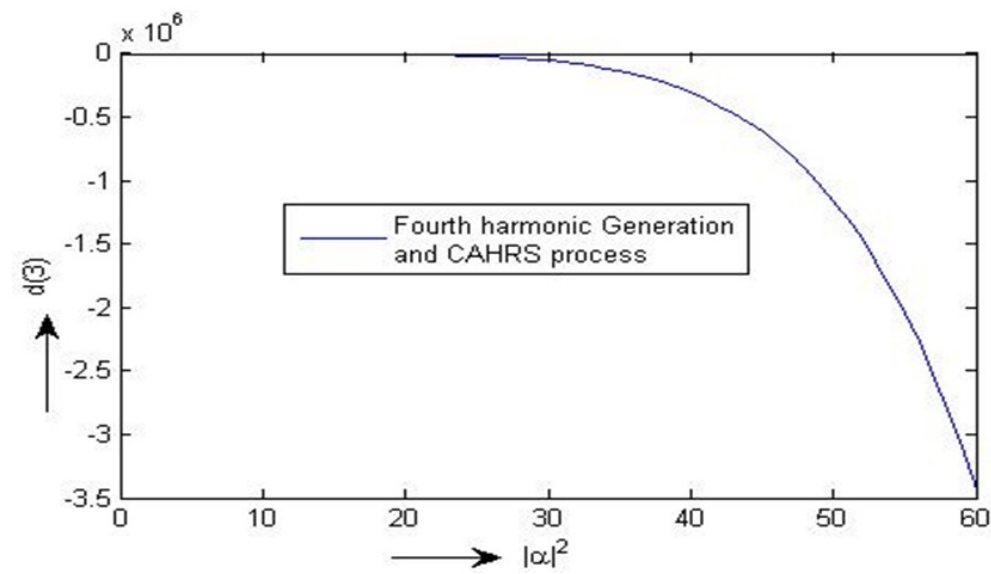

FIG. 1. The plot of third order photon antibunching $d(3)$ versus $|\alpha|^{2}$ in fourth harmonic generation and coherent anti-Stokes hyper-Raman scattering process (CAHRS) (taking $g^{2} t^{2} \approx 10^{-6}$ )

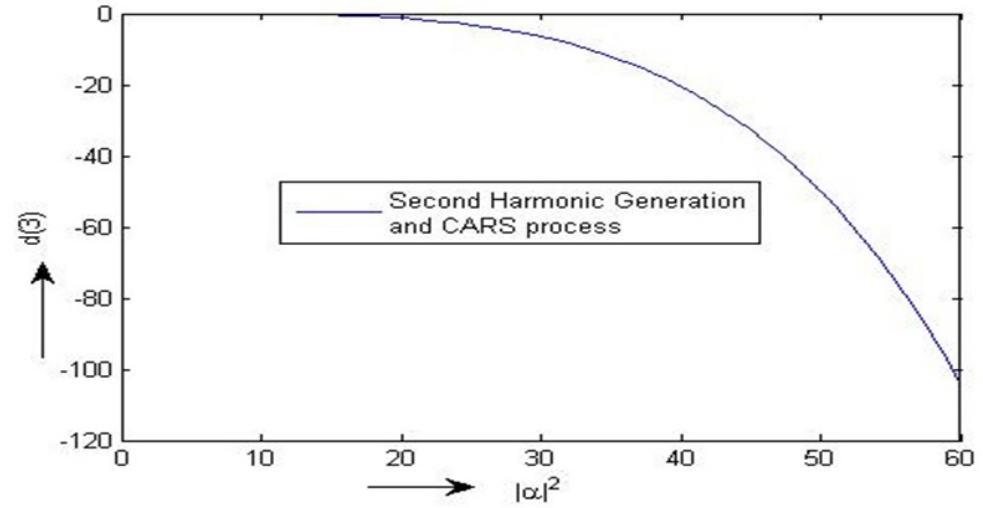

FIG. 2. The plot of third order photon antibunching $d(3)$ versus $|\alpha|^{2}$ in second harmonic generation and coherent anti-Stokes Raman scattering process (CARS) (taking $g^{2} t^{2} \approx 10^{-6}$ )

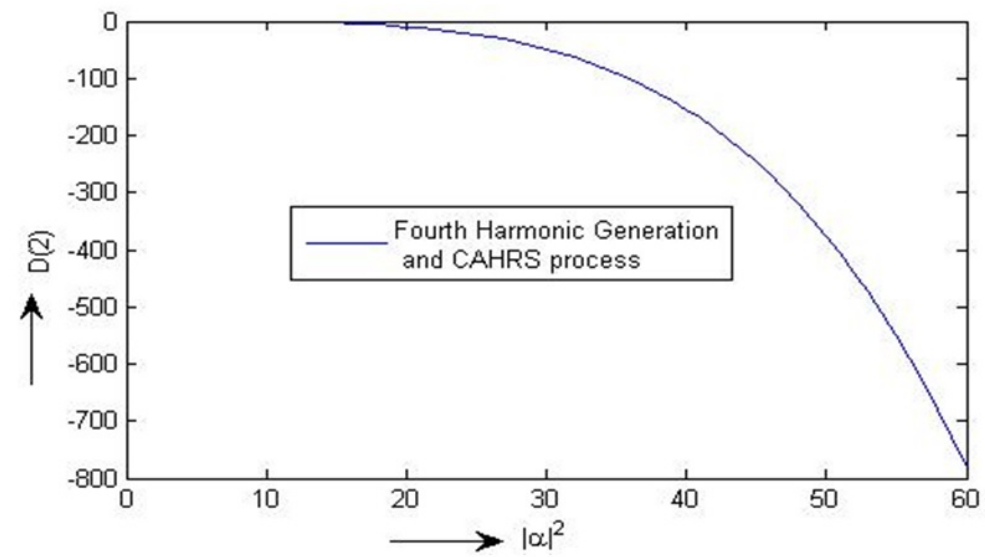

FIG. 3. The plot of higher order sub-Poissonian photon number statistics $D(2)$ versus $|\alpha|^{2}$ in fourth harmonic generation and coherent anti-Stokes hyper-Raman scattering process (CAHRS) (taking $g^{2} t^{2} \approx$ $\left.10^{-6}\right)$ 
TABLE 1. Results obtained for higher order photon antibunching (HOA) in non-linear optical processes

\begin{tabular}{|c|c|c|c|}
\hline $\begin{array}{c}\text { Sr. } \\
\text { no. }\end{array}$ & Optical processes & $\begin{array}{c}\text { Interaction } \\
\text { term }\end{array}$ & $\begin{array}{c}\text { Parameter } d(3) \\
\text { Expectation value w. r. t. *, } \\
|\alpha\rangle|0\rangle|0\rangle\end{array}$ \\
\hline 1 & $\begin{array}{c}\text { Second harmonic } \\
\text { generation }\end{array}$ & $A^{\dagger 2} B$ & $d(3)=-8 g^{2} t^{2}|\alpha|^{8}$ \\
\hline 2 & $\begin{array}{c}\text { Coherent anti-Stokes Raman } \\
\text { scattering (CARS) process }\end{array}$ & $A^{\dagger} B A^{\dagger} C$ & $d(3)=-8 g^{2} t^{2}|\alpha|^{8}$ \\
\hline 3 & $\begin{array}{c}\text { Fourth harmonic } \\
\text { generation }\end{array}$ & $A^{\dagger 4} B$ & $d(3)=-24 g^{2} t^{2}\left(3|\alpha|^{12}+4|\alpha|^{10}+|\alpha|^{8}\right)$ \\
\hline 4 & $\begin{array}{c}\text { Coherent anti-Stokes hyper-Raman } \\
\text { scattering (CAHRS) process }\end{array}$ & $A^{\dagger 2} B A^{\dagger 2} C$ & $d(3)=-24 g^{2} t^{2}\left(3|\alpha|^{12}+4|\alpha|^{10}+|\alpha|^{8}\right)$ \\
\hline
\end{tabular}

TABLE 2. Results obtained for higher order sub-Poissonian photon number statistics in non-linear optical processes

\begin{tabular}{|c|c|c|c|}
\hline $\begin{array}{c}\text { Sr. } \\
\text { no. }\end{array}$ & Optical processes & $\begin{array}{c}\text { Interaction } \\
\text { term }\end{array}$ & $\begin{array}{c}\text { Parameter } D(2) \\
\text { Expectation value w. r. t. } *, \\
|\alpha\rangle|0\rangle|0\rangle\end{array}$ \\
\hline 1 & $\begin{array}{c}\text { Second harmonic } \\
\text { generation }\end{array}$ & $A^{\dagger 2} B$ & $D(2)=-6 g^{2} t^{2}\left(|\alpha|^{4}\right)$ \\
\hline 2 & $\begin{array}{c}\text { Coherent anti-Stokes Raman } \\
\text { scattering (CARS) process }\end{array}$ & $A^{\dagger} B A^{\dagger} C$ & $D(2)=-6 g^{2} t^{2}\left(|\alpha|^{4}\right)$ \\
\hline 3 & $\begin{array}{c}\text { Fourth harmonic } \\
\text { generation }\end{array}$ & $A^{\dagger 4} B$ & $D(2)=-60 g^{2} t^{2}|\alpha|^{8}$ \\
\hline 4 & $\begin{array}{c}\text { Coherent anti-Stokes hyper-Raman } \\
\text { scattering (CAHRS) process }\end{array}$ & $A^{\dagger 2} B A^{\dagger 2} C$ & $D(2)=-60 g^{2} t^{2}|\alpha|^{8}$ \\
\hline
\end{tabular}

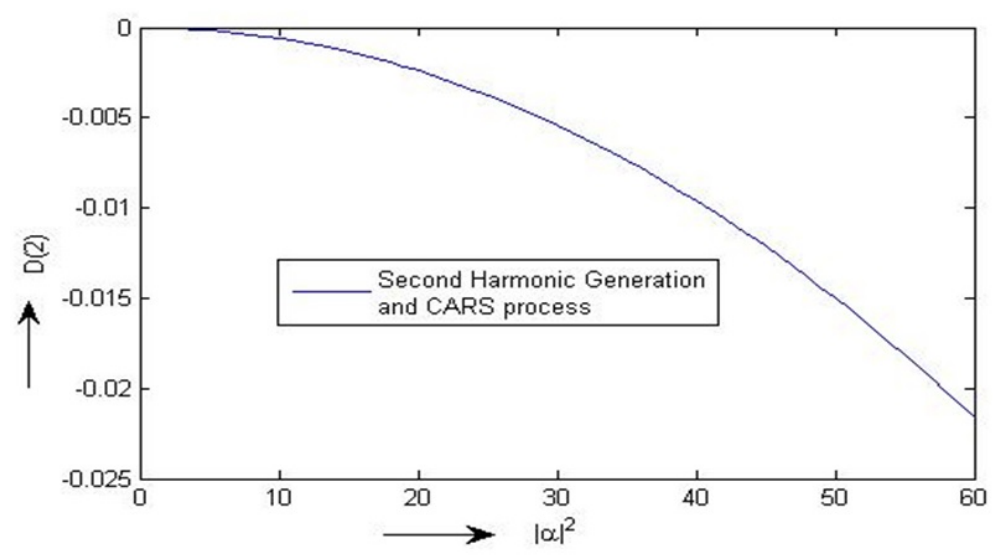

FIG. 4. The plot of higher order sub-Poissonian photon number statistics $D(2)$ versus $|\alpha|^{2}$ in second harmonic generation and coherent anti-Stokes Raman scattering process (CARS) (taking $g^{2} t^{2} \approx 10^{-6}$ )

\section{Result}

Tables 1 and Tables 2 illustrate the existence of higher order sub-Poissonian photon number statistics (HOSPS) and higher order photon antibunching (HOA) in different non-linear processes. If we plot a graph connecting higher order photon antibunching (HOA) say $d(3)$ and higher order sub-Poissonian photon number statistics (HOSPS) say D(2) with photon number in pump mode A i.e. $|\alpha|^{2}$, it is obvious that higher order sub-Poissonian photon number statistics (HOSPS) and higher order photon antibunching (HOA) increase non-linearly as $|\alpha|^{2}$ increases. Fig. 1 of higher order photon antibunching (HOA) and Fig. 3 of higher order sub-Poissonian photon number statistics (HOSPS) of fourth harmonic generation and coherent anti-Stokes hyper-Raman scattering (CAHRS) show the same values of higher order photon antibunching (HOA) as well as higher order sub-Poissonian photon number statistics (HOSPS). Similarly, Fig. 2 and Fig. 4 of second harmonic generation and coherent anti-Stokes Raman scattering process (CARS) also show the same values of higher order photon antibunching (HOA) as well as higher order sub-Poissonian photon number statistics (HOSPS). In 
all these non-linear optical processes, we have observed higher order photon antibunching (HOA) and higher order subPoissonian photon number statistics (HOSPS) only with respect to a quantum state which is the product of a coherent state $|\alpha\rangle$ for pump mode A and vacuum state $|0\rangle$ for Stokes mode B and signal mode C.

\section{Conclusion}

Higher order non-classical effects i.e. higher order photon antibunching (HOA) and higher order sub-Poissonian photon number statistics (HOSPS) in pump mode have been observed in a variety of non-linear optical processes. We have found that the non-linear processes having the same number of pump photons present prior to interaction have the same value of higher order photon antibunching and higher order sub-Poissonian photon number statistics, which we have demonstrated using examples of fourth harmonic generation with coherent anti-Stokes hyper-Raman scattering (CAHRS) and second harmonic generation with coherent anti-Stokes Raman scattering process (CARS). Further, we are obtaining the maximum value of non-classicality in fourth harmonic generation and coherent anti-Stokes hyper-Raman scattering process (CAHRS), as it has the maximum number of pump photons as compared to other non-linear optical processes which we have taken into consideration. As a result, we can conclude that non-classicality of a system can be revealed by the number of pump photons present in the system prior to interaction irrespective of the non-linear process involved. To study the higher order non-classical effects in various non-linear optical processes, we need the non-linear materials having higher order non-linear susceptibility which can be studied for making optical modulators and higher harmonic generators.

\section{References}

[1] Glauber R.J. Coherent and incoherent states of the radiation field. Physical Review, 1963, 131(6), P. $2766-2788$.

[2] Sudarshan E.C.G. Equivalence of semi-classical and quantum mechanical descriptions of statistical light beams. Physical Review Letters, 1963, 10(7), P. 277-279.

[3] Alexanian M. Non-classicality criteria: Glauber-Sudarshan P function and Mandel parameter. Journal of Modern Optics, 2017, 65(1), P. 16-22.

[4] Miranowicz A., Bartkowiak M., Wang X., Liu Y.X., Nori F. Testing non classicality in multimode fields: A unified derivation of classical inequalities. Physical Review A, 2010, 82(1), 013824(1-14).

[5] Alam N., Verma A., Pathak A. Higher-order non-classicalities of finite dimensional coherent states: A comparative study. Physics Letters A, 2018, 382(28), P. 1842-1851.

[6] Pathak A., Garcia M.E. Control of higher order antibunching. Applied Physics B, 2006, 84(3), P. $479-484$.

[7] Verma A. Study of higher order squeezing and sub-Poissonian photon statistics in generalized binomial state of light, International Conference on Photonics. Meta materials \& Plasmonics AIP Conference Proceeding, 2019, 2136, 050009(14).

[8] Verma A., Pathak A. Generalized structure of higher order non-classicality. Physics Letters A, 2010, 374(8), P. 1009-1020.

[9] Priyanka, Gill S. Higher Order Squeezing in Pump Mode in Multi-wave Mixing Process, In book: Advances in Communication and Computational Technology. Select Proceedings of ICACCT, 2019, P. 149-162.

[10] Gill S. Non Classical Effect of Light in Stimulated Five Wave Mixing. International Journal of Research and Scientific Innovation, 2017, 4(6), P. 90-93.

[11] Priyanka, Gill S. Study of Nonclassicality in Fifth Harmonic Generation Nonlinear Optical Process. Nanosystems: Physics, Chemistry, Mathematics, 2021, 12(1), P. 65-72.

[12] Mendoza K.Z., Dey S., Hussin V. Generalized squeezed states. Physics Letters A, 2018, 382(47), P. 3369-3375.

[13] Avenhaus M., Laiho K., Chekhova M.V., Silberhorn C. Accessing higher order correlations in quantum optical states by time multiplexing. Physical Review Letters, 2010, 104(6), 063602(1-4).

[14] Allevi A., Olivares S., Bondani M. High-order photon-number correlations: a resource for characterization and applications of quantum states. International Journal of Quantum Information, 2012, 10(08), 1241003(1-8).

[15] Allevi A., Olivares S., Bondani M. Measuring high-order photon-number correlations in experiments with multimode pulsed quantum states. Physical Review A, 2012, 85(6), 063835(1-5).

[16] Peřina J., Michálek V. Haderka O. Higher-order sub-poissonian-like non classical fields: Theoretical and experimental comparison. Physical Review A, 2017, 96(3), 033852(1-7).

[17] Alam N., Thapliyal K., Pathak A., Sen B., Verma A., Mandal S. Lower-and higher-order non-classicality in a Bose-condensed opto mechanical-like system and a Fabry-Perot cavity with one movable mirror: squeezing, antibunching and entanglement. arXiv preprintarXiv, $2017,1708.03967$.

[18] Thapliyal K., Pathak A., Sen B., Peřina J. Higher-order non-classicalities in a codirectional nonlinear optical coupler: quantum entanglement, squeezing, and antibunching. Physical Review A, 2014, 90(1), 013808(1-10).

[19] Thapliyal K., Pathak A., Sen B., Perina J. Nonclassicality in non-degenerate hyper-Raman processes. arXiv preprint arXiv, $2017,1710.04456$.

[20] Thapliyal K., Samantray N.L., Banerji J., Pathak A. Comparison of lower order and higher order non-classicality in photon added and photon subtracted squeezed coherent states. Physics Letters A, 2017, 381(37), P. 3178-3187.

[21] Mollow B.R., Glauber R.J. Quantum Theory of Parametric Amplification. Physical Review, 1967, 160(5), P. 1076-1096.

[22] Bennett C.H., Shor P.W., Smolin J.A., Thapliyal A.V. Entanglement Assisted Classical Capacity of Noisy Quantum Channels. Physical Review Letters, 1999, 83(15), P. 3081-3084.

[23] Laurenza R., Lupo C., Lloyd S., Pirandola S. Dense coding capacity of a quantum channel. Physical Review Research, 2020, 2(2), 023023(1-5).

[24] Braunstein S.L., D’Ariano G.M., Milburn G.J., Sacchi M.F. Universal Teleportation with a Twist. Physical Review Letters, 2000, 84(15), P. 34863489.

[25] Bennett C.H., Brassard G., Mermin N.D. Quantum cryptography without Bell's theorem. Physical Review Letters, 1992, 68(5), P. 557-559.

[26] Kim M.S. et. al. Entanglement by a beam splitter: Non-classicality as a prerequisite for entanglement. Physical Review A, 2002, 65(3), 032323(1-7).

[27] Bachor H.A.A Guide to Experiments in Quantum Optics. Weinheim: Wiley VCH, 1998, Chapters 8 and 10.

[28] Vyas R., Wang C., Singh S. Homodyne detection for the enhancement of antibunching. Physical Review A, 1996, 54(3), P. 2391-2396.

[29] Erenso D., Vyas R., Singh S. Higher order sub-Poissonian photon statistics in terms of factorial moments. Journal of Optical Society B, 2002, 19(6), P. 1471-1475. 
[30] Basak S., Chandrasekar R. Passive optical wave guiding organic rectangular tubes: tube cutting, controlling light propagation distance and multiple optical outputs. Journal of Materials Chemistry C, 2013, 2(8), P. 1404-1408.

[31] Hu S., Melton C., Mukherjee D. A facile route for the synthesis of nanostructured oxides and hydroxides of cobalt using laser ablation synthesis in solution (LASIS). Physical Chemistry Chemical Physics, 2014, 16(43), P. 24034-24044.

[32] Udayabhaskar R., Ollakkan M.S., Karthikeyan B. Preparation, optical and non-linear optical power limiting properties of Cu, CuNi nanowires. Appled Physics Letters, 2014, 104(1), 013017(1-4).

[33] Emelyanov A.V., Khenkin M.V., Kazanskii A.G., Forsh P.A., Kashkarov P.K., Gecevicius, Mindaugas, Beresna, Martynas, Kazansky, Peter G. Femtosecond laser induced crystallization of hydrogenated amorphous silicon for photovoltaic applications. Thin Solid Films, 2014, 556(4), P. 410413.

[34] Tang C., Zheng Q., Zhu H., Wang L., Chen S.C., Ma E., Chen X. Two-photon absorption and optical power limiting properties of ladder-type tetra phenylene cored chromophores with different terminal groups. Journal of Materials Chemistry C, 2013, 1(9), P. 1771-1780.

[35] Jiang T., Qin G., Qin W., Zhou J. Passively Q-switched erbium-doped fiber laser based on gold nanorods. Optik, 2014, 125(19), P. 5789-5793.

[36] Krishnan S., Sandeep C.S.S., Philip R., et al. Two-photon assisted excited state absorption in multiferroic $\mathrm{YCrO}_{3}$, nanoparticles. Chemical Physics Letters, 2012, 529(5), P. 59-63.

[37] Ren P., Fan H., Wang X. Electrospun nanofibers of $\mathrm{ZnO} / \mathrm{BaTiO}_{3}$, heterostructures with enhanced photocatalytic activity. Catalysis Communications, 2012, 25(8), P. 32-35.

[38] Xie R.H. H andbook of advanced electronic and photonic materials and devices (H.S. Nalwa edition). Academic Press, New York, 2000, 9, P. 267-307.

[39] Xie R.H., Rao Q., Jensen L. Encyclopedia of nanoscience and nanotechnology (H.S. Nalwwa edition). American Scientific, Los Angeles, 2003.

[40] Stentz A.J., Boyd R. Handbook of Photonics (M.C. Gupta edition), CRC press, Boca Raton, 1997.

[41] Hanamura E. Rapid radiative decay and enhanced optical nonlinearity of excitons in a quantum well. Physical Review B, 1988, 38(2), P. $1228-1234$.

[42] Lee C.T. Higher order criteria for non-classical effects in photon statistics. Physical Review A. 1990, 41(3), P. 1721-1723.

[43] An N.B. Multimode higher-order antibunching and squeezing in trio coherent states. Journal Optics B: Quantum and Semiclassical Optics, 2002, 4(3), P. 222-227.

[44] Prakash H., Mishra D.K. Higher order sub-Poissonian photon statistics and their use in detection of Hong and Mandel squeezing and amplitude squared squeezing. Journal of Physics B: Atomic, Molecular and Optical Physics, 2006, 39(9), P. 2291-2297.

Submitted 10 January 2022; revised 22 January 2022; accepted 2 February 2022

\section{Information about the authors:}

Priyanka - Department of Applied Science, University Institute of Engineering and Technology, Kurukshetra 136119, India; Chauhan7101@gmail.com

Savita Gill - Department of Applied Science, University Institute of Engineering and Technology, Kurukshetra 136119, India; Savita2015@kuk.ac.in

Conflict of interest: the authors declare no conflict of interest. 